1 | 2004

NOVECENTO... E DINTORNI

Dire la guerre?

\title{
Le guerre di Gaddus
}

(Da Stendhal a Céline)

\section{Bartolo Anglani}

\section{OpenEdition}

Journals

Edizione digitale

URL: http://journals.openedition.org/cei/231

DOI: 10.4000/cei.231

ISSN: 2260-779X

Editore

UGA Éditions/Université Grenoble Alpes

\section{Edizione cartacea}

Data di pubblicazione: 15 novembre 2004

Paginazione: 39-53

ISBN: 978-2-84310-057-4

ISSN: 1770-9571

Notizia bibliografica digitale

Bartolo Anglani, «Le guerre di Gaddus», Cahiers d'études italiennes [Online], 1 | 2004, online dal 15 mai 2006, consultato il 27 mars 2021. URL: http://journals.openedition.org/cei/231 ; DOI: https://doi.org/ 10.4000/cei.231 


\title{
LE GUERRE DI GADDUS
}

\section{(Da Stendhal a Céline ${ }^{1}$ )}

\author{
Bartolo Anglani \\ Université de Bari
}

\begin{abstract}
La guerre, voyez-vous, Bardamu, par les moyens incomparables qu'elle nous donne pour éprouver les systèmes nerveux, agit à la manière d'un formidable révélateur de l'Esprit humain! Nous en avons pour des siècles à nous pencher, méditatifs, sur ces révélations pathologiques récentes, des siècles d'études passionnées... Avouons-le franchement... Nous ne faisions que soupçonner jusqu'ici les richesses émotives et spirituelles de l'homme! Mais à présent, grâce à la guerre, c'est fait...
\end{abstract}

Voyage au bout de la nuit.

Tutte le volte che rileggo il Giornale di guerra e di prigionia, cerco di immaginare che cosa avremmo pensato noi, "posteri ricercatori" (30 dicembre 1915; GGP, p. 514), se Carlo Emilio Gadda fosse morto alla fine della guerra o se, per qualsiasi motivo, non fosse diventato quel grande scrittore che conosciamo. Come l'avremmo letto, questo testo così

1. La sigla GGP rinvia a: Carlo Emilio Gadda, Giornale di guerra e di prigionia, a c. di D. Isella, in Saggi, giornali, favole e altri scritti, vol. II, a c. di C. Vela e al., Milano, Garzanti, 1992. Questo intervento rispetta sostanzialmente la forma in cui era stato preparato per il seminario, e si presenta reintegrato nelle parti soppresse nell'esposizione per rispettare $\mathrm{i}$ limiti di tempo, corretto qua e là di qualche svista e completato di alcune note. Mi limito perciò a render conto della bibliografia solo in questa nota preliminare, scusandomi con gli autori se non ho potuto considerare i loro contributi nel corso della stesura. S'intende che pagine relative al Giornale e ai temi guerreschi in Gadda si trovano in tutte le principali monografie dedicate al nostro scrittore, del cui elenco dunque facciamo grazia al lettore. Per quanto riguarda i saggi dedicati esplicitamente all'argomento, fra i più recenti (alcuni dei quali comparsi dopo la stesura di questo intervento) posso segnalare: Giulio Ungarelli, Il tenente G., la cattura, la prigionia, in Le carte militari di G., Milano, Scheiwiller, 1994, p. 9-25; Guglielmo Gorni, G., o il testamento del capitano, in AA. VV., Le lingue di G., a c. di A.M. Terzoli, Roma, Salerno, 1995, p. 149-78; Marziano Guglielminetti, Gadda/Gaddus: il "Giornale di guerra e di prigionia» [1994], in Dalla parte dell'io. Modi e forme della scrittura autobiografica nel Novecento, Napoli, ESI, 2002, p. 251-65; Andrea Cortellessa, Il Duca di Sant'Aquila e la guerra degli altri. C.E. G. recensore di guerra, "Paragone - Letteratura", 46, n. 548-550, pp. 116-36; Id, I "De Officiis" dell'aspirante "valentuomo". La biblioteca 'militare' di G., in AA. VV., La biblioteca di Don Gonzalo. Il Fondo G. alla biblioteca del Burcardo, 2, a c. di A. Cortellessa e G. Patrizi, Roma, Bulzoni, 2001, p. 193-205; Maria Antonietta Terzoli, L'anima si governa per alfabeti. Note su G. scrittore di guerra, «Paragone - Letteratura», 49, n. 636-640, 2003, pp. 98-120; Christophe Mileschi, "La guerra è cozzo di energie spirituali»: estetica ed estetizzazione della guerra in C.E. G., «Bollettino '900», Electronic Newsletter of '900 Italian Literature, giugno 2003 (www2.unibo. it/boll900). 


\section{BARTOLO ANGLANi}

difficile da catalogare nella pur vasta fenomenologia della letteratura di guerra? Probabilmente avremmo schedato l'autore nella categoria dei memorialisti balzani, nevrotici, ossessivi, matti, complessati; oppure avremmo concluso che quel sottotenente degli alpini possedeva alcune qualità letterarie ma che a causa del suo carattere difficilmente sarebbe arrivato a farsi un nome nel mondo delle lettere. Ci saremmo chiesti forse anche come mai un figlio della borghesia milanese si firmava "Duca di Sant'Aquila" pur senza avere nel sangue alcun quarto di nobiltà; o come mai alternava questo appellativo aristocratico a quello maccheronico di "Gaddus»: e contemporaneamente ci saremmo meravigliati di trovare lui, duca di Sant'Aquila o Gaddus che fosse, così fortemente ed eticamente impegnato nella guerra. Dubbi e curiosità che richiamo solo in chiave paradossale, per sottolineare la qualità eccezionale di un libro che non può essere letto senza tener presente il destino futuro dell'autore.

A chiarire molti dubbi è intervenuto qualche anno fa un volume di Giancarlo Roscioni, dedicato all'infanzia e alla giovinezza del nostro autore e intitolato appunto Il Duca di Sant'Aquila. Rinvio a questo libro affascinante, al quale sono debitore di molte osservazioni che mi capiterà di fare nel corso di questa conversazione ${ }^{2}$. Da Roscioni apprendiamo che il soprannome di Duca di Sant'Aquila era stato scelto da Gadda bambino quando, nei giochi con il fratello Enrico e con la sorella Clara, il giardino e i dintorni della casa di campagna erano stati divisi in ducati e marchesati, e il piccolo Carlo Emilio si era insignorito del Ducato di Sant'Aquila. Giochi d'infanzia attraverso i quali siamo passati tutti, anche se non tutti con la sfrenata fantasia salgariana del piccolo Carlo Emilio. E infatti noi, persone più o meno normali, crescendo abbandoniamo i nostri giochi $\mathrm{e}$ diventiamo grandi, e non ci verrebbe mai l'idea di scrivere sulla nostra carta di visita i titoli di cui ci siamo insigniti in gioventù ${ }^{3}$.

Gadda invece, arruolatosi come volontario nella guerra del 1915 e nominato sottotenente degli alpini, arriva a Edolo in Valle Camonica dove è stato destinato dai superiori, si reca al Bazar, acquista un quaderno, annota scrupolosamente sul verso della copertina: "Acquistai questo qua-

2. Voir Gian Carlo Roscioni, Il Duca di Sant'Aquila. Infanzia e giovinezza di G., Milano, Mondadori, 1997.

3. Molti anni dopo (nel 1963), a chi durante un'intervista gli domandava per qual ragione nei diari di guerra comparisse il nome del duca di Sant'Aquila, Gadda spiegò la cosa come « un gioco, o meglio un sogno fanciullesco", e ricordò che "ducato e contea erano due ciliegi eretti nel vento, strapazzati dalle crude raffiche del tramontano ", lamentandosi però che il diario fosse stato "pubblicato "tel quel" " senza che all'autore fosse stata data "la possibilità di rivedere e correggere lo scritto". (Intervista televisiva per il Prix International de Littérature 1963, in "Per favore mi lasci nell'ombra". Interviste 1950-1972, a c. di C. Vela, Milano, Adelphi, 1993, p. 88.) 
derno oggi, in Edolo, al Bazar Edolo. 24 agosto 1915. CE Gadda», sulla copertina scrive "Gaddus. - 1915», comincia a registrare i fatti che gli accadono nei primi giorni del servizio e poi, arrivato al 2 settembre, stende un pezzo burlesco che comincia così :

Hodie quel vecchio Gaddus e Duca Sant'Aquila arrancò du' ore per via sulle spallacce del monte Faetto, uno scioccolone verde per castani, prati e conifere, come dicono i botanici, e io lo dico perché di lontano guerciamente non distinsi se larici o abeti vedessi. (GGP, p. 452)

A questo punto il lettore ha un sobbalzo : è comparso il grande Gadda, il Gadda del pastiche, dell'ironia e del comico-tragico : ma il lettore lo riconosce perché già lo conosce, e perché prima ancora di leggere il Giornale ha letto almeno La cognizione del dolore e Il Pasticciaccio. E il lettore si chiede quale relazione ci sia tra questo burlesco Gaddus Duca di Sant'Aquila e il sottotenente Carlo Emilio Gadda che spinto da profondo senso del dovere si è arruolato volontario e, in attesa di compiere azioni eroiche, per il momento svolge con scrupolo quasi maniacale le proprie mansioni militari.

Qualche giorno prima, il 27 agosto, Gadda ha ricordato le «pazze scritture», lettere infarcite di scherzi burleschi e maccheronici che egli suole scambiarsi con un amico, ed ha osservato che se un giorno quelle lettere divenissero pubbliche "potrebbero sembrar miserabili rispetto al tempo in cui furono scritte", dal momento che in esse "si esprimono solo quei sentimenti che la lontananza vieta di altrimenti manifestare, solo quelle sciocchezze che allegrano talora la nostra antica conversazione»; e che in esse non si contiene "tutta la nostra vita, tutto il nostro animo", ed anzi «la parte migliore dei nostri sentimenti vi è quasi estranea, come se adombrasse di venir tratta ad accompagnare cose meno alte» (GGP, p. 448). Con questa avvertenza Gadda sta predisponendo una specie di batteria difensiva; sta cioè preparando il terreno per disinnescare (uso di proposito una terminologia militare, dato il contesto) il potenziale esplosivo della scrittura maccheronica e burlesca, relegandola in un angolo minore senza tuttavia rinunciare ad essa: le pazze scritture sono solo un divertimento e non hanno niente a che fare con la serietà tragica del «tempo". Excusatio non petita, si potrebbe dire, o negazione freudiana che è la stessa cosa. Perché il passo citato, scritto qualche giorno dopo, mostra le pazze scritture intrise del dolore e del male del " tempo » : tanto è vero che qui si racconta uno "scherzo" dell'immaginazione che «tragicamente» viene interrotto da "un rumore subitaneo ", dal « rimbombo lontano della cannonata "(2 settembre 1915; GGP, p. 453). È un passo che dovrebbe esser letto per intero, perché il suo senso sta non nelle affermazioni singole ma nelle variazioni e nei trapassi del registro, del linguaggio, delle tonalità. Solo 


\section{BARTOLO ANGLANI}

così si può comprendere quanto è forte e inestricabile in Gadda l'intreccio fra dolore e gioco, fra esperienza esistenziale ed esperienza estetica. Gadda si lascia andare all' «immaginare», ma la sua immaginazione è alimentata dall'angoscia del presente e si riflette su di essa. Il grande Gadda è nato.

Questa miscela di eroismo e di letteratura, di sentimento fortissimo della propria realtà individuale e del gioco di pseudonimi, di vocazione bellica e di tentazione immaginaria, di immedesimazione e di estraniazione (se si esclude la vocazione al pastiche), richiama un nome che certo non è il primo a venire in mente a proposito di Gadda: Stendhal. E non dico ciò solo per omaggio a Grenoble. C’è qualcosa di stendhaliano nel giovane Gadda, che funziona come un fattore fondativo del suo rapporto con la vita e con la letteratura. Va da sé che non parlo della "chasse au bonheur", che in Gadda assume apparenze goffe e patetiche. È vero che anche Stendhal visse le proprie esperienze erotiche e sentimentali in forme a volte impacciate e piene di gaffes (come dimenticare le «lunettes vertes" ?), e patì a volte qualche fiasco, ma nessuno può negare che la sua vita (come quella dei suoi principali eroi) fu piena di rapporti d'ogni genere fino quasi all'ultimo giorno. Gadda sperimenta invece qui tutta la propria inettitudine sentimentale e la registra impietosamente. Come quando racconta di aver incontrato " una graziosissima cameriera del lago di Garda, dai folti capelli castani, altissima, snella», e di essersi proposto «di farle la corte» a dispetto degli altri "cinque colleghi» che si trovano nell'albergo; poi di aver detto "quattro galanterie, male impastate per la stanchezza ", alla cameriera, la quale "gentilmente le accettò " (2 settembre 1915; GGP, p. 452). La sventurata rispose? Non credo : penso che la corte sia terminata qui. Anche se Gadda persevera nel cercare altre avventure: "Andai ieri a trovare una bella ragazza, certa Giuditta Giorgi, che mi diede appuntamento per oggi. Non so se ci andrò ", scrive il 14 ottobre 1915 (GGP, p. 474). "Limonai anche con una sarta qui dirimpetto", annota il 9 novembre 1915 (GGP, p. 489), e il giorno dopo: "Qui dirimpetto ce sta una bella sarta di 17 anni; quasi le farei fare delle camice; ma ho paura che pasticci» (ibidem). Seguono altri conati, quasi sempre patetici e goffi:

La cameriera dell'albergo Derna, a cui volevo far la corte, mi pare insipida e in complesso m'attira mediocremente. - Mi piace di più la vivandiera del reggimento, per quanto meno bella, e una cameriera dell'Hôtel Edolo, a cui diedi un appuntamento per domenica scorsa, che mancai per andare in montagna. Lo rinnovai per oggi: speriamo che ella ci sia. (12 settembre 1915; GGP, p. 462)

Purtroppo la ragazza «mancò all'appuntamento", scrive tre giorni dopo Gadda, il quale, «non essendo più in tempo a prendere la via della montagna", "bighellonò per il paese, "pieno di cattivo sole» (ibidem). Quando va in un paesino alla ricerca di "qualche femina permeabile», egli 
si accorge che purtroppo il paese è «deserto" e se ne torna "pian piano e tranquillo» a Edolo (2 novembre 1915; GGP, p. 485). Gli unici rapporti andati a buon fine sono quelli a pagamento. Finché esplode con la dichiarazione solenne e tragica che egli non rispetterà mai «l'obbligo di presentare una fidanzata", perché "le difficoltà finanziarie» e "la debolezza» della sua volontà lo lasceranno "sempre imbarazzato" (25 settembre 1916; GGP, p. 629). Ma con questa affermazione siamo già dentro il grande lavoro di autoanalisi che Gadda intraprende nel suo diario, e ci tocca tornare indietro e riprendere il discorso daccapo. Limitiamoci a ricordare che mai più, nell'opera gaddiana, troveremo riferimenti diretti a una propria attività sentimentale. Con la guerra e con la prigionia, la vita erotica di Gadda sembra chiusa per sempre.

Ma torniamo agli aspetti in cui invece si può parlare di 'stendhalismo' in un senso più proprio. Non sono in grado di affermare decisamente se, prima di andare in guerra, Gadda avesse letto Stendhal. Del resto, come si sa, le letture gaddiane non sono spesso documentabili. «Posso dire che ho letto pochissimi libri in vita mia", affermò in una intervista a Dacia Maraini (1968), con una punta di snobismo e di provocazione ${ }^{4}$. È del resto molto difficile appurare quando Gadda abbia "effettivamente» avuto « tra le mani» $\mathrm{i}$ « romanzi del grandi narratori stranieri (Balzac, Zola, Dickens, Dostoevskij, ecc.) menzionati nei quaderni di guerra o in scritti dei primi anni Venti ${ }^{5}$ ». Dunque non è importante stabilire per via filologica se prima della guerra egli avesse letto Il Rosso e il Nero o La Certosa di Parma. Nel suo diario ricorre almeno due volte il termine "egotismo", ma- come si vedrà - in accezione fortemente negativa ${ }^{6}$. Se avesse potuto vedere gli scritti autobiografici di Stendhal, probabilmente Gadda sarebbe stato colpito dall'uso dei croquis, assai simili agli schizzi che egli disseminò nel suo diario ${ }^{7}$. Ma non sono questi gli aspetti decisivi. Con Stendhal, o

4. Gadda, «Per favore, mi lasci nell'ombra» cit., p. 172.

5. Roscioni, Il Duca di San'Aquila cit., p. 91.

6. L'atteggiamento cambia in testimonianze più tarde. Nel 1949, nel saggio Emilio e Narcisso, Gadda discute con competenza il termine "egotismo»: «Lo Stendhal è un egotista: e sa di essere, oltre tutto. Non si rizza insino all'altitudine del Nano, del Nano in trono, dell'Ajaccio : non si protubera da podio, nella volgarità priapata del Predappio: non arriva all'egotismo di un musico, o di un poeta in genere, o di un poeta lirico in ispecie: del divo di Pescara: ma egotistuccio gli è lui pure, se pur prosatore.» (Carlo Emilio Gadda, I viaggi la morte, in Saggi giornali favole e altri scritti. I, a c. di L. Orlando e al., Milano, Garzanti, 1991, p. 640). In un saggio del 1953 (L'egoista) Gadda aggiunge che Stendhal «sembra aver intuito la relazione di concomitanza giovinezza-egotismo" e commenta che "da quel genio che era" Stendhal aveva capito "che una qualche differenza ci doveva essere fra egoismo ed egotismo" (ivi, p. 659).

7. Giulio Ungarelli indica un punto ancora più rilevante nella 'poetica' narrativa che prescrive «la estrema fedeltà alla verità», per quanto "diseroica o bassa possa essere», aggiungendo che "la testimonianza 'storica' " di Gadda sembra "aver fatto tesoro della lezione stendhaliana di Fabrizio del Dongo alla battaglia di Waterloo" (Ungarelli, Il tenente G. cit., p. 19). 


\section{BARTOLO ANGLANi}

meglio con qualche personaggio stendhaliano, Gadda condivide il desiderio di compiere qualche azione eroica, e al tempo stesso la persuasione amara di essere nato in un tempo sbagliato. Quando per il Giornale parla di «estetismo militaresco e stendhaliano», di «vitalismo» e di «spirito avventuroso e 'stendhaliano' ", Roscioni non solo si rifa ad un'atmosfera, ad una pulsione che può manifestarsi come frutto del tempo (D'Annunzio è sempre molto vicino) senza essere necessariamente prodotta da letture e da modelli rivissuti consapevolmente, tanto è vero che sùbito dopo invita $\mathrm{i}$ lettori a "fare i conti con la poliedricità e la contraddittorietà » dello spirito gaddiano, contrassegnato da «impulsi rigoristici, quasi ascetici » e da " esigenze tecnico-funzionali» del tutto assenti (soprattutto i primi, come si sa), dallo spirito stendhaliano ${ }^{8}$ : ma cita un passo di Gadda medesimo, il quale in uno scritto autobiografico intitolato Abbozzi di temi per tesi di laurea confessò di aver "cercato ed amato la vita militare in guerra" per la sua "'passione' estetica, simbolistica, verso gli atti e le operazioni militari», e ammise che il gioco era "finito male» per lui che aveva "pagato una multa umiliante, giusto castigo di questo estetismo militaresco e stendhaliano ${ }^{9} "$.

Filologicamente accertabile è invece la presenza di Stendhal nel Gadda postbellico, e soprattutto in quel Cahier d'études steso fra il 1924 e il 1925 (dopo il ritorno dal soggiorno argentino) e pubblicato solo nel 1983 con il titolo di Racconto italiano di ignoto del Novecento: e non solo perché nell'Elenco delle letture da fare per la redazione del romanzo (datato 26 marzo 1924) Gadda al terzo punto scrive: «Rouge et Noir: (Stendhal) richiami di espressione ${ }^{10}$ ", ma soprattutto perché egli intende attribuire al giovane fascista protagonista del romanzo progettato, Grifonetto Lampugnani, alcuni tratti stendhaliani. Il giovanotto, come Julien Sorel e Fabrizio del Dongo, «non ha fatto la guerra perché ragazzo »:

È un ipervolitivo (Gatti, Rouge et Noir) : studio, ambiente intellettuale, mancanza del padre, non grande ricchezza. (Inserire forse qualche cosa del Rouge et Noir) (Forse no, perché l'epilogo potrebbe essere tale da far credere a una copia di Rouge et Noir) ${ }^{11}$.

E nell'epilogo avrebbe dovuto uccidere la donna di cui era innamorato:

Egli la uccide? O l'aveva uccisa prima dopo il colloquio? O era morta? Bisogna chiarire bene questo punto e non cascare nell'esagerato o nel poliziesco. Vedere eventualmente il Trionfo della Morte del D'Annunzio o lo Stendhal in Rosso e nero ${ }^{12}$.

8. Roscioni, Il Duca di San'Aquila cit., p. 125.

9. Il passo, appartenente a un inedito gaddiano, è citato da Roscioni, ibidem.

10. Carlo Emilio Gadda, Racconto italiano di ignoto del novecento (Cahier d'études), in Scritti vari e postumi, a c. di A. Silvestri e al., Milano, Garzanti, 1993, p. 573.

11. Ivi, p. 398.

12. Ivi, p. 400 . 
Più che questi riferimenti diretti, però, l'aura stendhaliana del romanzo è evidente nell'intreccio di vitalismo e di idealismo che caratterizza il personaggio nel quale Gadda si specchia (benché, ad onor suo, nel suo fascismo giovanile mai egli compì non dico omicidi ma nessuno degli atti teppistici da lui attribuiti a Grifonetto), e che risalta nella premessa, là dove si legge che è «meglio giocare una volta un gioco disperato che vivere inutilmente la tragica, inutile vita ${ }^{13}$ ", e in altri luoghi apertamente autobiografici: "ho bisogno di provarmi, di vedere quello che posso fare ${ }^{14}$.

Di Stendhal e dei personaggi stendhaliani il giovane Gadda possiede la vocazione all'autoanalisi, e stendhalianamente scrive con il proprio diario un romanzo di educazione alla fine del quale l'eroe rimane sconfitto. Come i più grandi personaggi di Stendhal, infine, Gadda vive immerso nel mondo reale e risente in forme acutissime della contingenza storica; e insieme abita un mondo sotterraneo impermeabile alla storia, dominato da una pulsione tragica alla ripetizione e alla morte.

Come Fabrizio del Dongo, Gadda va in guerra per due motivi. Il primo viene confessato e sbandierato per tutto il diario, ed è il desiderio di compiere atti eroici e di conquistare la gloria. Il secondo, nascosto ma ben presente alla mente dell'autore, può essere ricostruito dalla lettura: è il desiderio di morte. Non di una morte eroica, che come tale non può non essere messa in conto da chi cerca la gloria, ma la morte in se stessa, ovvero la voglia di annullarsi e di non esistere. Non importa che Fabrizio del Dongo partecipi alla guerra da esterno, indossando una divisa abusiva, mentre Gadda è regolarmente arruolato. Il volontario Gadda (lodato dai superiori per disciplina, ordine, capacità intellettuali e pratiche) resta in realtà estraneo al vero mondo militare: non lo comprende mai per davvero, ne vede gli aspetti grotteschi e ridicoli e ne patisce tutta l'irrazionalità. Non si integra mai. Dopo la sconfitta di Caporetto tenta di ricostruire i fatti con grande puntigliosità, aiutandosi come sempre con gli schizzi e le mappe, ma non riesce a capire che cosa sia davvero accaduto. La dinamica generale della battaglia gli rimane estranea: proprio come era avvenuto a Fabrizio del Dongo sulla scena di Waterloo. Scrive infatti:

Avrei desiderato sapere come si svolgeva l'attacco, a che punto i nemici erano giunti. Ma nulla si vedeva, notizie non ne arrivavano, e colpi di fucile non ne sentii, e nemmeno ne sentirono i miei soldati. (Memoriale, cap. 22; GGP, p. 717)

\section{E ancora :}


Cercavamo con inquietudine il sottostante bosco con lo sguardo, ma la nebbia ci permetteva di scorgere i primi alberi soltanto.» (Memoriale, cap. 24; GGP, p. 719)

Potremmo estendere questa metafora della "nebbia» a tutta l'esperienza militare gaddiana: il narratore del diario vive una storia complessa e tragica senza veramente comprenderla.

Dunque, «la morte sul campo è bella, mille volte preferibile alla morte nel letto ", afferma Gadda (9 settembre 1915; GGP, p.461). Egli soffre «dell'inazione» quando ancora non è al fronte (22 settembre 1915; GGP, p. 469). Vorrebbe combattere. La frequenza della parola «dovere» ricorda l'ossessione con cui Julien Sorel si ripete di dover compiere un «devoir». Ma a questo desiderio di gloria non corrispondono né le circostanze esteriori né, soprattutto, quelle interiori. Sempre come un personaggio stendhaliano, Gadda sa di vivere nel tempo sbagliato. Per Julien Sorel il tempo dell'eroismo era quello di Napoleone; per Gadda il tempo dell'eroismo è stato quello del Risorgimento. Egli credeva di partecipare ad una "Guerra per l'Indipendenza", come scrive sul frontespizio del diario del 1916 (GGP, p. 527), e invece si trova coinvolto in un'avventura tragicomica, tanto più tragica quanto più la realtà è ìmpari rispetto ai grandi modelli del passato ed alle necessità del presente. "Ho bell'e visto che ben difficilmente potrò distinguermi in questa guerra: sono capitato in un punto morto", osserva il 18 luglio del 1916 (GGP, p. 569), e forse ancora non si rende conto che con l'espressione "un punto morto " non ha indicato solo il luogo fisico particolare in cui è capitato, ma la condizione generale del suo operare. Da un lato, il mondo è inadatto alla produzione di atti eroici. «La mia patria mi è lontana; la vita pantanosa della caserma, e di una caserma simile, annega in me le gioie e gli entusiasmi che mi potrebbero venire dalla contemplazione della grande storia presente, mi fa scordare le speranze, mi prostra, mi attutisce il desiderio di sagrificio ", scrive il 23 settembre del 1915. Ma, sùbito dopo, vede se stesso «debole come il più debole degli uomini, gettato in una vita orribilmente tormentata a questi giorni di squallore spirituale», ed esclama: «Se qualche cosa di eroico sorgesse in me!» E prima ci aveva fatto credere che qualche cosa di eroico lo possedeva già. In realtà non gli manca «il desiderio di combattere», ma esso "si ottunde nei disappunti, nelle controversie, nel veleno della vita fangosa di questi giorni». Ed ecco allora che «i giorni dell'infanzia, della adolescenza» tornano a torturarlo "con visioni di felicità perduta» (ivi, p. 471).

L'esistenza eroica è minacciata, dunque, sia dalla realtà esterna fangosa e pantanosa, sia però anche dall'inettitudine del soggetto. Da un lato, la stessa Patria e lo stesso popolo, per i quali è andato in guerra, gli appaiono 
«alla prova ben peggiori» di quanto avesse creduto ( 2 novembre 1915; $G G P$, p. 486): tutto nell'esercito, dalla truppa fino alle più alte sfere, è disordine e caos. Non è solo la polemica contro i "generaloni» inetti (15 settembre 1915 ; GGP, p. 464), contro "quelle mucche gravide, quegli acquosi pancioni di ministri e di senatori e di direttori e di generaloni» (20 settembre 1915; GGP, p. 467), perché questa polemica è abbastanza diffusa in quel tempo: basti ricordare il romanzo di Lussu $U n$ anno sull'altipiano per comprendere di che pasta fossero fatti coloro che guidarono la guerra nel 1915-1918 (e non solo quella). Il fatto è che Gadda comincia a lodare il soldato semplice, vittima dei generaloni e dei profittatori di guerra, come quando scrive che «i nostri uomini sono calzati in modo da far pietà ", con "scarpe di cuoio scadente e troppo fresco per l'uso, cucite con filo leggero da abiti anzi che con spago, a macchina invece che a mano ", con quel che segue, e conclude che i soldati "portano il vero peso della guerra, peso morale, finanziario, corporale, e sono i peggio trattati» (GGP, p. 466-7) : ma alle prime difficoltà vede gli stessi soldati come "muli bastardi" che devono essere costretti a fare "tutto il loro dovere, senza misericordia» (6 ottobre 1915; GGP, p. 473), molto più "pigri » degli austriaci (22 giugno 1916; GGP, p. 543). Il soldato italiano non è del tutto cattivo, ma "è pigro, specie il meridionale: è sporchetto per necessità, come il nemico, ma anche per incuria: provvede ai bisogni del corpo nelle vicinanze della trincea, riempiendo di merda tutto il terreno: non si cura di creare un unico cesso; ma fa della linea tutto un cesso ", poi "dormicchia durante il giorno" anche se «in compenso però è paziente, sobrio, generoso, buono, soccorrevole, coraggioso, e impetuoso nell'attacco» (22 giugno 1916; GGP, p. 546). Del resto, osserva Gadda con compiacimento, gli alpini che si trovano ai suoi ordini «hanno uno spirito più elevato anche perché si infonde loro un po' del mio" (25 giugno 1916; GGP, p. 547): eppure tutti, appena possono, "piagnucolano artriti, dolori, indigestioni, ecc. : alcuni a ragione, altri schifosamente, per fifa» (13 luglio 1916; GGP, p. 566). Il sottotenente Gadda può anche infischiarsi dei superiori nel suo privato e limitarsi a salutarli con deferenza, ma non tollera che altrettanto facciano i subordinati :

L'italiano, nel momento della fatica, brontola, e se la prende coi superiori: ed è ingiusto: e io dico allora: italiano carogna. (9 giugno 1916; GGP, p. 537)

Ma l'inettitudine e il disordine sono anche interni, radicati nell'animo del sottotenente degli alpini. Gadda vorrebbe compiere qualche azione eroica ma sa o sospetta o teme di non esserne del tutto capace. Il male non è solo esterno, dunque, ma si annida nell'interno dell'anima. E così, per decifrare l'enigma di se stesso, Gadda avvia nel diario un'analisi acutis- 
sima, dolorosa, protratta di sé. Bisogna ammettere che non aspetta di avere i cinquant'anni di Stendhal per chiedersi : chi sono io? e per iniziare a conoscersi. Ma, proprio come un eroe stendhaliano, scopre la natura di se stesso riflettendo sulle sue reazioni alle 'prove' della vita. E la guerra è una prova suprema e senza appello. Il difetto più grave che egli riscontra in se stesso è la mancanza di volontà :

La volontà non è temprata bene; onde il mio spirito non sa procacciarsi quella serenità e quella calma che sono l'edificio più nobile cui possa pervenire un uomo. Ondeggio tra un desiderio e

l'altro; tra un sogno e l'altro. (15 ottobre 1915; GGP, p. 475)

«Ma io devo e voglio combattere», proclama qualche giorno dopo (27 ottobre 1915; GGP, p.481), riconoscendo però che la sua "sensibilità morbosa» lo ostacola (2 novembre 1915; GGP, p. 486) e che in fondo egli è " un povero essere" (7 novembre 1915; GGP, p. 488), dal momento che la degradazione dei tempi non impedisce agli altri, ad altri soldati, di compiere "episodî eroici" (18 novembre 1915; GGP, p. 494) di una "grandezza omerica» (18 novembre 1915; GGP, p. 495). Per questo egli precipita sempre più in una terribile "crisi spirituale" (25 novembre 1915 , $G G P$, p. 497). Purtroppo non posso citare e commentare i numerosi passi in cui via via Gadda prosegue la sua autoanalisi : come quello in cui si definisce "un organismo procul sentiens» (ivi, GGP, p. 500), o quello in cui registra «uno incorreggibile squilibrio fra la realtà empirica e l'apprezzamento che il [suo] essere ne fa in relazione con le necessità della sua esistenza», e che «implica la sufficienza nel comprendere ma l'insufficienza nell'agire, oltre che nel volere» (28 novembre 1915; GGP, p. 503). Come Julien Sorel, Gadda avverte la sproporzione fra la sua sensibilità e la sua intelligenza, da un lato, e la "paralisi volitiva" dall'altro (17 dicembre 1915; GGP, p. 507) : e, come Julien Sorel che si costringe a stringere la mano di Madame de Rênal ${ }^{15}$, tenta di trasformarsi con lo sforzo della volontà in un essere volitivo ed eroico, e spera che il suo «sistema nervoso, viziato congenitamente da una sensività morbile, sostenga, grazie allo sforzo cosciente dell'animo, l'orrore della guerra", di una guerra che " ancora e sempre e non per ostinazione polemica e non per indifferenza di 'imboscato'" egli crede "necessaria e santa" (5 giugno 1916; GGP, p. 533). Usare la volontà per trasformarsi in essere volitivo è un bel paradosso: volere di volere... Ed è, naturalmente, un segno di impotenza. Egli però ce la mette tutta. Ma quando gli càpita l'occasione di sottoporsi a una

15. «Julien, indigné de sa lâcheté, se dit: Au moment précis où dix heures sonneront, j'exécuterai ce que, pendant toute la journée, je me suis promis de faire ce soir, ou je monterai chez moi me brûler la cervelle». (Stendhal, Le Rouge et le Noir. Chronique du XIX" siècle, Paris, Gallimard, 1940, p. 81). 
"prova», ossia a un allarme aereo, sente di "non esser molto coraggioso" perché tali allarmi destano in lui «una lieve apprensione»: e tuttavia, "per un senso di fierezza" verso se stesso, fa tutto ciò che deve fare "con la massima calma", gira "per la città", attende alle sue faccende "come nulla fosse» (14 giugno 1916; GGP, p. 540).

Attraverso l'autoanalisi, però, Gadda arriva ad un esito che i personaggi stendhaliani, e Stendhal stesso, si erano ben guardati dal raggiungere: all'odio per l'Io. Così egli rovescia l'egotismo in una malattia terribile dell'individuo e della società. Il male sta proprio nel voler essere, ciascuno, un Io. Il disordine e il pasticcio del mondo, che in un primo tempo Gadda attribuisce al solo carattere degli italiani, sono prodotti dall'elefantiasi dell'Io. Dapprima, Gadda svolge osservazioni sparse, di carattere moralistico-sociologico, sulla propensione degli italiani a «litigare per sciocchezze e con sterilità di risultati ». Egli vorrebbe avere "sempre, ovunque, affabilità, cortesia, assenza di egotismo nelle discussioni e anche uno zinzino di fantasia e di ragionevolezza", e invece "tutto questo bociare a vanvera " gli provoca "noia, e talora una rabbia» (25 agosto 1915; GGP, p. 447). Ben presto Gadda si rende conto che «l'egoismo personale è l'unica legge di molti " (6 ottobre 1916; GGP, p. 472). Ma con queste osservazioni siamo ancora nel regno delle genericità, delle frasi comuni a ogni tempo e ogni luogo sull'egoismo umano. Ma il 24 luglio del 1916 Gadda esplode, e unifica i termini delle sue riflessioni in un impasto personalissimo in cui è già tutto il Gadda futuro, quello della Cognizione del dolore. L'elemento essenziale del carattere dei "porchi italiani» è il disordine:

Quand'è che i miei luridi compatrioti di tutte le classi, di tutti i ceti impareranno a tener ordinato il proprio tavolino da lavoro? a non ammonticchiarvi le carte d'ufficio insieme alle lettere della mantenuta, insieme al cestino della merenda, insieme al ritratto della propria nipotina, insieme al giornale, insieme all'ultimo romanzo, all'orario delle Ferrovie, alle ricevute del calzolaio, alla carta per pulirsi il culo, al cappello sgocciolante, alle forbici delle unghie, al portafogli privato, al calendario fantasia? Quando, quando? Quand'è che questa razza di maiali, di porci, di esseri capaci soltanto di imbruttire il mondo col disordine e con la prolissità dei loro atti sconclusionati, proverrà alle attitudini dell'ideatore e del costruttore, sarà capace di dare al seguito delle proprie azioni un legame logico?

E questo disordine è a sua volta espressione di una natura più profonda, della paura per il proprio Io:

Combattere tra soldati che hanno paura d'una fucilata, che ingialliscono al rumore del cannone nemico, che se la fanno addosso al pensiero d'un pericolo lontano, e non perché hanno moglie e figli (non raccontatemi mai una tal balla) ma solo per paura personale, paura di me, paura di io, paura di esso Io, del proprio Io, del proprio Io-me, combattere tra questi, come sono due o tre dei miei giannizzeri, che il diavolo li scoglioni, che gusto è?

La conclusione è che, benché l'eroismo non manchi, c'è purtroppo anche il disordine: "quello c'è, sempre, dovunque, presso tutti: oh! se c'è, 


\section{BARTOLO ANGLANi}

e quale orrendo, logorante disordine! Esso è il mare di Sargassi per la nostra nave» (24 luglio 1916; GGP, p. 574). L'eroismo e il volontarismo sono messi al servizio di un principio collettivo più alto. A differenza degli eroi stendhaliani, i quali dopo aver scontato la discrepanza tra aspirazioni eroiche e realtà degradata concentrano ogni sforzo alla promozione (e alla distruzione) dell'io, per Gadda la percezione della degradazione contemporanea accentua sempre più la sproporzione fra il primato etico della "logica associativa» e l'«incoercibile narcisismo», l'«incoercibile egoismo" degli italiani ${ }^{16}$, dando prova di quelle "antinomie mostruose racchiuse nella falsa unità della persona " che egli considera caratteristiche di sé ${ }^{17}$.

Ma questo diarista, così angosciato dalla prepotenza dell'Io-me e dall'irrazionalità del mondo, è capace di sentimenti estetici improvvisi e violenti. Anche per questo aspetto mi viene in mente una sequenza stendhaliana: quella in cui Julien Sorel, entrando nella stanza del tribunale di Besançon per essere processato e condannato a morte, resta «frappé de l'élégance de l'architecture ${ }^{18} »$. Incapace di comprendere la ragione degli avvenimenti, Gadda 'descrive' le cose con minuziosità e, descrivendole, le trasforma in oggetti di esperienza estetica. Non posso che accennare brevemente a questa tematica: si va dal piacere provato per una «meravigliosa giornata autunnale", per "le più dolci tinte, i più dolci giorni, i più dolci monti, tenerissime nebbie e sole» (26 ottobre 1915; GGP, p. 478), alla vista della "pineta di Val Canaglia tersissima e nera contro il cielo lucido" (31 agosto 1916; GGP, p. 601), al «bel sole d'ottobre» che "riempie le ondulazioni dell'Altipiano, vaporanti di nebbia» (4 ottobre 1916; GGP, p.635), fino ai "varî aspetti pittorici della guerra» che gli piacerebbe "poter ricordare», come "monti spelacchiati dal bombardamento» o "prati pieni di buche di granate e di trincee campali» o "lo spettacolo degli accampamenti nelle pinete» con "uomini intirizziti che si scaldano al fuoco rosso dei pini nell'oscurità mattinale e serale» (18 ottobre 1916; GGP, p. 641) : fino alla «fila di soldati neri» della giornata di Caporetto, che Gadda all'inizio scambia per italiani e invece sono tedeschi (Memoriale, cap. 31 ; GGP, p. 733). E tutto questo a dispetto delle " condizioni difficili », materiali e spirituali, in cui il diario viene scritto, "male, in fretta, sotto lo sguardo dei curiosi che allungano l'occhio, ti chiedono,

16. Carlo Emilio Gadda, Gli italiani sono democratici? [1963], in "Per favore mi lasci nell'ombra" cit., p. 18.

17. Gadda, Racconto italiano cit., p. 177.

18. Stendhal, Le Rouge et le Noir cit., p. 484. 
fantasticano, fanno supposizioni ${ }^{19}$ ". La ragione di questo pardosso, che trasforma un diario composto giorno per giorno durante manovre e battaglie in un testo provvisto di una forte valenza estetica, sta nel fatto che quel diario è un diario solo formalmente e tende in realtà allo statuto dell'autobiografia. Fatto del quale l'autore sembra consapevole quando a Dacia Maraini, la quale nel 1968 gli chiede se abbia mai pensato di scrivere un'autobiografia, risponde che le sue «memorie di guerra sono un'autobiografia ${ }^{20}$ ». La sfumatura è essenziale. Nel diario gli avvenimenti si succedono indipendentemente da quella volontà ricostruttiva dell'autore che invece nell'autobiografia ordina e regola la struttura del racconto. Ebbene, la struttura narrativa e significativa del Giornale è così compatta da suscitare nel lettore ignaro il sospetto che non di un vero diario si tratti ma di un finto diario scritto tutto con piena consapevolezza letteraria a partire dal punto d'arrivo già stabilito. Ciò che dà al diario un'allure autobiografica, fin dall'inizio, è la consapevolezza di partecipare a una "tragedia comune, politica ed etnica» e "morale», scatenata dalle "mattane degli uomini ", alla quale l'autore ha "partecipato intensamente" (31 dicembre 1915; GGP, p. 516). La sindrome della sconfitta, e il correlativo senso di colpa per la sconfitta stessa, sono iscritti ab initio nel mondo gaddiano. Gadda scrive il diario, giorno per giorno, come se già conoscesse Caporetto, la sua Waterloo irredimibile ${ }^{21}$.

Così questo libro bellissimo e straziante, che deve essere considerato il vero archetipo del mondo gaddiano, a dispetto di ciò che la storia racconta (e cioè che la guerra del 1915-18 fu vinta dall'Italia e dalle forze alleate contro gli Imperi Centrali), documenta in termini estetici e conoscitivi altissimi l'esperienza bruciante e indelebile di una sconfitta. Tale convinzione non dipende solo dall'ottica di chi, costretto in prigionia, assisté non da protagonista all'evolversi della situazione bellica e ricevé la fine della guerra non come una conquista ma come un regalo immeritato alla propria viltà, ma anzi accompagna Gadda fino alle estreme testimonianze della sua vita. Un trauma insanabile impedisce al reduce di accettare l'idea che dopo la sua Waterloo c'è stata la ripresa e la battaglia di Vittorio Veneto. Nel Castello di Udine della guerra si scrive sempre come di una guerra perduta: e particolarmente nel saggio Impossibilità di un diario di guerra, dove l'autore argomenta, appunto, sulla impossibilità di scrivere

19. Intervista a Cesare Garboli (1967), in "Per favore mi lasci nell'ombra» cit., p. 134.

20. Intervista cit. a Dacia Maraini, ivi, p. 166.

21. Agiscono dunque, nel giovane Gadda, "una ipotesi di conoscenza e una ricerca di azione, di prassi, intrinsecamente disposte alla sconfitta" (Giuseppe Bonifacino, Il groviglio delle parvenze. Studio su C.E. G., Bari, Palomar, 2002, p. 15). 
un diario di guerra alludendo antifrasticamente al diario già scritto e, a quell'epoca, tenuto segreto. Né i lettori dell' "Ambrosiano » (dove il saggio uscì la prima volta nel 1931), né quelli del Castello (pubblicato nel 1934) possedevano il privilegio di noi, "posteri ricercatori", di poter risalire al Giornale per trovare la fondazione irreversibile dell'universo gaddiano e la radice ossessiva di quelle che potevano allora apparire come osservazioni o divagazioni sparse. Tutte le pagine successive di Gadda, e non solo quelle direttamente connesse al tema bellico, porteranno l'impronta di ciò che durante la guerra l'autore ha scoperto, non solo su di sé ma sulla logica stessa del mondo. Egli ha visto la stendhaliana «volontà " giovanile «sommersa dal caso, come una barca dalla risacca, e «il chiaro pensiero onnubilarsi e dissolversi nella stanchezza ${ }^{22}$ ». Per sempre Gadda porterà nella coscienza il rimorso di non esser morto a tempo:

Tutto sommato, date le premesse, io dovevo rimanerci : e sarebbe stata la cosa più logica, la sola cosa logica e degna. Non esserci rimasto significa indubbiamente aver abdicato alla verità, nell'incerto presagio di un qualche presumibile rubinetto. Essere era disparire: sopravvivere significò non essere ${ }^{23}$.

Non conta nulla che la guerra sia stata vinta, perché l'ottica ormai non è più quella storica ma quella metafisicamente negativa del "non essere». "Non credo più alle vittorie, ogni vittoria in guerra è una vittoria di Pirro ", dirà Gadda a Corrado Stajano nel $1968^{24}$. Sul piano del giudizio storico egli potrà rivedere $\mathrm{i}$ suoi giudizi e persino arrivare a definire Giolitti - l'odiato politicante contro il quale era sceso in piazza nel 1915"un uomo probo" che "forse aveva ragione ${ }^{25}$ ", ma sul piano esistenziale egli resterà pur sempre colui che, simbolicamente, "aveva letto e sofferto " la narrazione zoliana della Débâcle ${ }^{26}$. Non che Zola fosse divenuto il suo modello ideologico e narrativo: ché nella rappresentazione della guerra come una meccanica insensata, raccontabile solo in termini straniati e crudeli, pur senza averne coscienza Gadda era passato dal modello stendhaliano all'ombra di quel Céline che lo aveva "preceduto nell'impostazione narrativa e stilistica ${ }^{27}$ ». Solo molti anni dopo, leggendo il Voyage, Gadda si accorgerà delle affinità elettive esistenti fra il suo mondo e quello céliniano. Non importa che Gadda si fosse arruolato sull'onda di un vitalismo razionale, convinto eticamente della "necessità della guerra ${ }^{28}$,

22. Carlo Emilio Gadda, Il Castello di Udine, Torino, Einaudi, 1971, p. 30.

23. Carlo Emilio Gadda, Dal castello di Udine verso i monti [1931], in Il castello di Udine cit., p. 52.

24. Gadda, "Per favore mi lasci nell'ombra" cit., p. 177.

25. Intervista del $1972 ; i v i$, p. 218.

26. Intervista del 1967; ivi, p. 127.

27. Intervista del 1972 ; ivi, p. 213.

28. Gadda, Impossibilità cit., p. 38. 
mentre Céline (sotto la maschera sottile del suo personaggio) ci era arrivato per caso, da perfetto "anarchiste» con la testa poco "solide» e senza rendersene conto, mentre la guerra si avvicinava "sans qu'on s'en soye rendu compte ${ }^{29}$ »: ché, a guardar bene sotto le prime apparenze, il «bond d'enthousiasme» che per un attimo suscita il gesto di «héroïsme» del giovane Ferdinand ${ }^{30}$ esprime una carica non molto diversa da quella che aveva spinto il giovane Carlo Emilio a 'provare' se stesso. Dolorosamente e contraddittoriamente condiviso con un fine patriottico quello di Gadda, e irresponsabilmente irrazionale quello di Céline: ma figure entrambi di un vitalismo di sconfitti, di grotteschi nietzschiani destinati a rimanere 'al di qua' dell'uomo-eroe. Da prospettive opposte, sperimentando l'assurdità e l'orrore della guerra, essi si impegnano a demolire stilisticamente, con deformazioni grottesche, la retorica e il «mito» stesso della guerra ${ }^{31}$, lasciandosi dominare da una perversa e dolorosa voluttà di profanazione. I "generaloni " gaddiani non sono poi molto diversi da quelli céliniani, così come non molto diverso è lo spettacolo di vigliaccheria, di ignoranza e di superba improntitudine, di corruzione e di incapacità che i due eserciti (e, dunque, i due popoli) dànno nella rappresentazione dei nostri due scrittori. Con il Giornale, testo che secondo l'autore non bisognava chiamare "opera» ma "solo testimonianza e confessione», raccolta di "frammenti d'un diario occasionale e incompiuto ${ }^{32}$ ", Gadda, partito dalla stendhaliana volontà di potenza, ha iniziato il suo lungo viaggio verso la notte.

29. Louis-Ferdinand Céline, Voyage au bout de la nuit, Paris, Gallimard, 1952, p. 8-9.

30. Ivi, p. 10.

31. Gadda, Elogio di alcuni valentuomini [1931], in Il castello di Udine cit., p. 24.

32. Carlo Emilio Gadda, A proposito del "Giornale di guerra e di prigionia" [1965], in "Per favore mi lasci nell'ombra» cit., p. 124. 